\title{
Diversidade de insetos antófilos em áreas com reflorestamento de eucalipto, Município de Triunfo, Rio Grande do Sul, Brasil ${ }^{1}$
}

\author{
Letícia Azambuja Lopes², Betina Blochtein ${ }^{3} \&$ Ana Paula Ott ${ }^{4}$
}

1. Parte da Dissertação de Mestrado da autora sênior, PPG-Zoologia, Pontifícia Universidade Católica do Rio Grande do Sul.

2. Laboratório de Entomologia, PUCRS, Caixa Postal 1429, 90619-900 Porto Alegre, RS, Brasil. (leazambuja@gmail.com)

3. Faculdade de Biociências, Pontifícia Universidade Católica do Rio Grande do Sul, Av. Ipiranga, 6681, 90619-900 Porto Alegre, RS, Brasil. (betinabl@pucrs.br)

4. Faculdade de Agronomia, Departamento de Fitossanidade, Universidade Federal do Rio Grande do Sul, Av. Bento Gonçalves, 7712 , 90012-970 Porto Alegre, RS, Brasil. (ana.ott@ufrgs.br)

\begin{abstract}
Diversity of anthophile insects in an area with eucalypt plantations in the municipality of Triunfo, Rio Grande do Sul, Brazil. The result of habitat fragmentation has been assessed through measuring of richness and diversity of some functional groups, especially flower-visiting insects. These organisms allow functional connectivity between plantations and protected areas and the restoration of disturbed environments. This study aimed to analyze the diversity of anthophilous insects on transects established between a gallery forest and the eucalyptus reforestation and exclusively among a section of eucalyptus trees in south Brazil. Insects collected belonged to the orders Hymenoptera, Lepidoptera, Diptera, Coleoptera and Hemiptera. Insect abundance was low compared to other studies. Twenty-eight species were found in both environments, whereas 138 were restricted to one environment (61 in gallery forest/eucalyptus, and 77 in eucalyptus/eucalyptus). Insects visited flowers of 31 plants species distributed in 20 families. In both environments, there were a few dominant species and a large number represented by a single individual. A similarly high diversity was found in both environments $\left(\mathrm{H}^{\prime}=4.084\right.$ and $\left.\mathrm{H}^{\prime}=4.018\right)$. Among insects, bees showed the highest diversity in environments gallery forest/eucalyptus $\left(\mathrm{H}^{\prime}=3.211\right)$ and eucalyptus/eucalyptus $\left(\mathrm{H}^{\prime}=2.8\right)$. Despite the similarity in community structure between these environments, their fauna of anthophilous insects differ in species composition and the number of individuals of those shared species.
\end{abstract}

KEYWORDS. Floral visitors, bees, abundance, gallery forest, Eucalyptus spp.

RESUMO. O efeito da fragmentação de habitats tem sido avaliado medindo-se a riqueza e diversidade de determinados grupos funcionais, especialmente insetos visitantes florais. Esses organismos possibilitam conectividade funcional entre plantações e áreas protegidas e a restauração de ambientes alterados. Este estudo objetivou analisar a diversidade de insetos antófilos ao longo de transectos estabelecidos entre mata ciliar e reflorestamento de eucaliptos e exclusivamente entre talhões de eucaliptos, no sul do Brasil. Os insetos coligidos pertencem às ordens Hymenoptera, Lepidoptera, Diptera, Coleoptera e Hemiptera. Comparativamente a outros estudos, a abundância de insetos foi baixa. Vinte e oito espécies foram encontradas em ambos ambientes, enquanto 138 foram exclusivas em um ambiente (61 em mata ciliar/eucaliptos e 77 em eucaliptos/eucaliptos). Os insetos visitaram flores de 31 espécies vegetais, representantes de 20 famílias. Nos dois ambientes foram encontradas poucas espécies dominantes e um grande número representadas por um único indivíduo. A alta diversidade foi similar nos dois ambientes de estudo $\left(\mathrm{H}^{\prime}=4,084\right.$ e $\left.\mathrm{H}^{\prime}=4,018\right)$. Entre os insetos, as abelhas mostraram a maior diversidade nos ambientes mata ciliar/eucaliptos $\left(\mathrm{H}^{\prime}=3,211\right)$ e eucaliptos/eucaliptos $\left(\mathrm{H}^{\prime}=2,8\right)$. Considerando-se a similaridade na estrutura da comunidade entre os ambientes, a fauna de insetos antófilos difere quanto à composição de espécies e número de indivíduos das espécies amostradas.

PALAVRAS-CHAVE. Visitantes florais, abelhas, abundância, mata ciliar, Eucalyptus spp.

A conservação da biodiversidade representa um dos maiores desafios enfrentados atualmente, em função dos elevados níveis de perturbações antrópicas dos ecossistemas. Uma das principais consequiências dessas perturbações é a fragmentação de paisagens que reduz significativamente o fluxo de animais, pólen e sementes (Aizen \& Feinsinger, 1994; Kruess \& TscharntKe, 1994; Samways, 1995; Kearns \& Inouye, 1997; Pickett et al., 1997; VIANA \& PINHEIRO, 1998).

O efeito da fragmentação de habitats tem sido avaliado medindo-se a riqueza e diversidade de determinados grupos funcionais, especialmente de invertebrados (KREMEN et al., 1993). Dentre estes, os artrópodos correspondem a cerca de $75 \%$ dos animais na terra, dos quais $89 \%$ são insetos, com seis milhões de espécies estimadas (SpeIGHT et al., 1999). Os insetos apresentam ampla distribuição geográfica e adaptações relacionadas a diferentes habitats e hábitos alimentares (JoLIVET, 1992; SAMWAYs, 1995), por isso mostramse como excelente grupo para evidenciar mudanças em ecossistemas (SAMWAYS, 1995).
A exemplo de insetos antófilos, determinadas espécies especialistas parecem ser mais suscetíveis à fragmentação de habitats do que espécies generalistas. Numerosas espécies são estreitamente relacionadas a determinadas plantas como fontes de alimento (néctar, pólen ou óleos florais) ou substrato para a reprodução (local para acasalamento, construção de ninhos e oviposição) (DiDHAm et al., 1996; BROWN JR., 1997; BROWN JR. \& FREITAS, 2002). Em contrapartida, espécies vegetais dependem de interações com os visitantes florais como polinizadores (KeARNS et al., 1998; SPEIGHT et al., 1999). A dinâmica dos ecossistemas é influenciada pela diversidade de insetos antófilos de tal modo que cerca de $70 \%$ das plantas com flores depende destes organismos para sua polinização (TEPEdino, 1979; SEASTEDT \& CRossley, 1984).

Dentre os atributos conferidos às florestas tropicais está a elevada biodiversidade, devida aos numerosos nichos ecológicos e à complexidade das relações dentro das cadeias alimentares e entre os componentes das 
diferentes comunidades. Por outro lado, nas áreas florestadas, que ao longo do tempo são submetidas a diversas formas de manejo para fins específicos, a diversidade e a abundância das formas de vida são determinadas pela amplitude de nichos adequados para sua sobrevivência. Assim, a biodiversidade em talhões homogêneos de espécies florestais é obviamente mais baixa do que em áreas naturais altamente diversificadas (Poggiani \& Oliveira, 1998).

Espécies de Eucalyptus representam grande parte da área reflorestada no mundo devido a sua capacidade de adaptação a diferentes habitats, ao rápido crescimento e ampla aplicabilidade de sua madeira (OliveIra et al., 2001; SANTOs et al., 2002; Pinto et al., 2004). No Brasil, a eucaliptocultura ocupa cerca de 2.954 .780 ha de área plantada e proporciona a redução da pressão exaustiva sobre as reservas nativas. Porém, o efeito alopático de metabólitos do eucalipto inibe fortemente o desenvolvimento de plantas herbáceas (Del Moral \& MulLer, 1969; Souto et al., 2001). Assim, a implantação destas florestas altera amplamente os habitats naturais e implica na perda da biodiversidade local (PogGiani \& Oliveira, 1998; Viana \& Pinheiro, 1998). Com relação à entomofauna, plantios de eucalipto causam impactos significativos sobre a diversidade, riqueza e distribuição das espécies (MAJER \& RECHER, 1999).

Mundialmente a destruição de habitats é apontada como a principal ameaça à biodiversidade (IUCN, 2002) . FonTANA et al. (2003) apontam que este fator representa cerca de $42 \%$ da ameaça à fauna em extinção no Rio Grande do Sul. Na Depressão Central do Estado, na década de 60 , a mata foi substituída pela agricultura e plantações de acácia e eucalipto (RAmBO, 1994). A cobertura original dessa região é de elementos originários da Floresta Estacional Decidual, cujo conceito ecológico está ligado ao clima (TeiXeIRA et al., 1986).

A recomposição de habitats alterados depende da conectividade funcional (como dispersão) com áreas conservadas (TSCHARNTKE et al., 2002). A biodiversidade terrestre é totalmente dominada por insetos, refletida pelo total envolvimento deste grupo nos processos ecológicos deste ambiente (Hutcheson, 1999). Neste contexto, o consumo de materiais vivos das plantas faz dos insetos visitantes florais os principais polinizadores das plantas com flores (WASER, 1978).

A fitofisionomia da Depressão Central no Rio Grande do Sul, característica da confluência dos Rios Taquari e Jacuí, sofreu forte ação antrópica. Entretanto, o conhecimento sobre a fauna de insetos antófilos é restrito. Neste sentido, objetiva-se analisar a diversidade de insetos antófilos em dois ambientes com distinta ação antrópica no Horto Florestal AES-SUL, em Triunfo, RS.

\section{MATERIAL E MÉTODOS}

O Horto Florestal AES-SUL Ltda (29 $52^{\prime} 00^{\prime \prime} \mathrm{S}$ $29^{\circ} 56^{\prime} 00^{\prime \prime} \mathrm{S}$; 51 $1^{\circ} 45^{\prime} 00^{\prime \prime} \mathrm{W}-51^{\circ} 41^{\prime} 00^{\prime \prime} \mathrm{W}$ ) localiza-se no Distrito de Barreto, município de Triunfo, RS. Pertence à Zona Subtropical Sul de STRAHLER (1977), com média anual de temperatura de $19^{\circ} \mathrm{C}$, normais pluviométricas anuais superiores a $1800 \mathrm{~mm}$ e umidade relativa do ar superior a 70\% (FERRARo \& HASENACK, 2000). O horto possui área aproximada de 1542 ha, sendo a cobertura vegetal de 1043 ha composta por plantios de Eucalyptus alba, E. saligna, E. botrioides, E. tereticornis, E. citriodora, E. grandis e E. paniculata (Myrtacea). As espécies predominantes são E. tereticornis e E. citriodora. Os povoamentos de eucaliptos estão divididos em talhões de 0,5 a 40 ha que diferem quanto à idade $(\mathrm{n}=57 ; \overline{\mathrm{x}}=12,4 ; \mathrm{s}=13,5)$, área ocupada $(\mathrm{n}=57 ; \overline{\mathrm{x}}=8,2 ; \mathrm{s}=7,6)$ e altura $(\mathrm{n}=57 ; \overline{\mathrm{x}}=17 ; \mathrm{s}=$ 4,5) das árvores; os talhões estão separados por estradas de terra destinadas ao trânsito de caminhões. Nas margens do rio Taquari, em uma extensão aproximada de $5 \mathrm{~km}$, há uma área de 248 ha com remanescente de mata nativa. A área restante é ocupada por campos com diferentes graus de ação antrópica, estradas, banhados e açudes.

Os insetos antófilos foram amostrados em duas trilhas que representam situações características no local; em cada uma delas, foi percorrido um transecto de $250 \mathrm{~m}$ de comprimento e aproximadamente $6 \mathrm{~m}$ de largura. Uma trilha representa a transição entre o remanescente de mata ciliar e um talhão de eucalipto (Eucalyptus citriodora e E. tereticornis) e a outra localiza-se entre dois talhões de eucalipto (Eucalyptus alba, E. citriodora e E. tereticornis).

As coletas mensais ocorreram no período de setembro de 2002 a agosto de 2003, entre 9:00 e 16:00 h, contemplando as variações sazonais e temporais relacionadas aos visitantes florais. Os insetos foram coligidos diretamente em flores de plantas herbáceas e arbustivas, com auxílio de rede entomológica, seguindose metodologia proposta por SAKAGAMI et al. (1967). Os espécimes foram montados em alfinetes e conservados a seco na coleção de referência do Laboratório de Entomologia da PUCRS. A identificação dos insetos foi efetuada consultando-se a bibliografia especializada ou mediante a colaboração de especialistas.

Para a avaliação do tamanho amostral empregado, utilizou-se o método de rarefação segundo KREBS (1998). Além de estimar o tamanho amostral, a rarefação serve como medida de diversidade, uma vez que permite a comparação da variação da riqueza de espécies com o número de indivíduos coletados (BUDDLE, 2001).

A diversidade da entomofauna de visitantes florais foi avaliada utilizando-se os índices de Shannon ( $\left.\mathrm{H}^{\prime}\right)$ usando-se o logaritmo natural $(\operatorname{logn})$, Simpson $(\lambda)$, e Equitabilidade de Pielou (J'), aplicados aos registros de abundância relativa das espécies, de acordo com MAGURRAN (1988).

A constância de insetos, que demonstra a relação entre a proporção dos dias em que a espécie $i$ foi coletada e o número total de dias amostrados, foi categorizada conforme SiLVEIRA Neto et al. (1976): constante > 50\%, acessória $>25-50 \%$ e acidental $<25 \%$. A dominância das espécies foi definida de acordo com as categorias estabelecidas de FRIEBE (1983), sendo eudominante > 10\%, dominante $>5-10 \%$, subdominante $>2-5 \%$, recessiva $=1-$ $2 \%$ e rara $<1 \%$. D\% = (i/t).100, onde i é o total de indivíduos de uma espécie e t o total de indivíduos coletados.

Para identificar a estrutura da comunidade, foram testados modelos matemáticos que tomam como base a curva de distribuição da abundância proporcional das espécies. Para tanto, foram utilizados os programas 
Biodiversity Professional Beta 1.0 versão 1.0 (MCALEECE, 1997) e Past (Hammer \& Harper, 2003). A similaridade entre as áreas foi medida pelos índices de similaridade de Sorensen (S) para dados qualitativos (MAGURRAN, 1988).

Os dados climáticos foram obtidos no $8^{\circ}$ Distrito de Meteorologia do Instituto Nacional de Meteorologia (INMET) e utilizados para análise da correlação entre a abundância de insetos com a temperatura e precipitação, calculada através da Correlação de Pearson (r), processada com o programa SPSS.

\section{RESULTADOS E DISCUSSÃO}

Abundância da entomofauna antófila. Durante o estudo foi registrada a ocorrência de 504 indivíduos de 148 espécies de insetos visitantes florais, pertencentes a cinco ordens (Tab. I). Dentre os táxons coletados, a maior abundância ocorreu em Hymenoptera (276) e Lepidoptera (134), seguida de Diptera (64), Coleoptera (25) e Hemiptera (5) (Fig. 1).

O reduzido número de exemplares amostrados, comparativamente à Silveira Neto et al. (1995), CHEY et al. (1998) e Hingston \& PotTs (1998), parece estar relacionado a diferentes metodologias de coleta e características ambientais. Segundo Silveira Neto et al. (1995), o impacto ambiental decorrente de atividades agrícolas determinou redução significativa no número de indivíduos e táxons amostrados em São Paulo.

No Horto Florestal AES-SUL, a baixa abundância de insetos visitantes florais pode ser atribuída, principalmente, às condições de conservação deste ambiente. Del Moral \& Muller (1969) e Huttel \& Loumeto (2001) destacam o empobrecimento da vegetação rasteira determinado pelas plantações de eucaliptos. Segundo Morales \& Arzen (2002), distúrbios no habitat podem alterar as interações entre insetos antófilos e plantas devido à influência da composição e abundância das flores. Estes autores comentam que este processo é mais aparente quando o distúrbio está associado à invasão de plantas exóticas, que representa abundante recurso de néctar e pólen tanto para espécies de visitantes florais nativas quanto introduzidas.

A composição da fauna de insetos antófilos

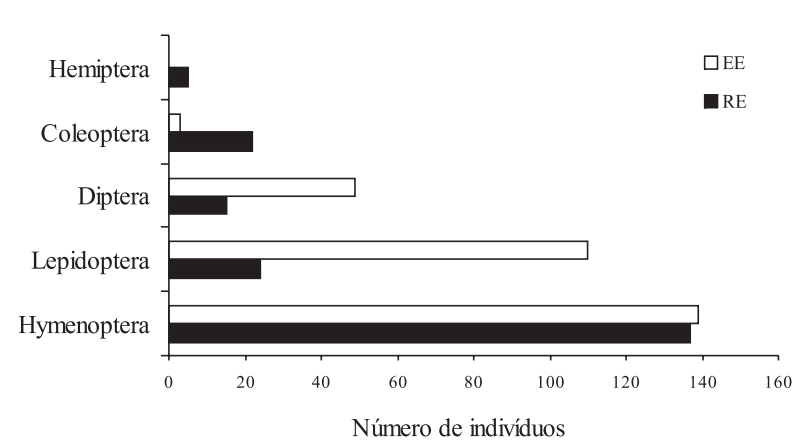

Fig. 1. Representatividade dos táxons de insetos antófilos coletados no período de setembro de 2002 a agosto de 2003, no Horto Florestal AES-SUL, Triunfo, RS. (RE, trilha entre remanescente de mata ciliar e talhão de eucalipto; EE, trilha entre dois talhões de eucalipto). registrada neste estudo foi, provavelmente, influenciada pela atratividade das flores de eucaliptos que competem com o estrato herbáceo-arbustivo. Em populações naturais na Tasmânia, Eucalyptus globulus foi visitado por 71 espécies de insetos antófilos (Hingston \& PotTs, 1998). Segundo LARA (1992), outro fator que interfere na amostragem dos insetos é a luminosidade, pois é limitante para a periodicidade de vôo dos insetos. Durante este estudo, observou-se que o sombreamento produzido pelo estrato arbóreo do eucalipto, principalmente após as 14 horas, reduziu a atividade dos insetos nas flores. Após este horário coletou-se apenas $4 \%$ do total de insetos amostrados.

Os grupos de insetos antófilos encontrados no Horto Florestal AES-SUL corroboram com os registrados para outros locais, sendo a maior representatividade de Hymenoptera, Lepidoptera e Diptera (Bosch et al., 1997; Wilms et al., 1997, COMBA et al., 1999; Pascarella et al., 2001; Morales \& Aizen, 2002). De modo semelhante, estas três ordens também são prevalecentes em amostragens amplas de entomofauna (MARINONI \& DUTRA, 1991; CHEY et al., 1998).

$\mathrm{Na}$ trilha entre o remanescente de mata ciliar e o talhão de eucalipto foram registrados 203 insetos visitantes florais: Hymenoptera (68\%), Lepidoptera (12\%), Coleoptera (11\%), Diptera (7\%) e Hemiptera (2\%). Na trilha entre dois talhões de eucalipto, não houve registro de hemípteros e os 301 indivíduos amostrados pertencem às ordens Hymenoptera (46\%), Lepidoptera (37\%), Diptera (16\%) e Coleoptera (1\%). Dentre as espécies registradas, vinte e oito foram comuns às duas áreas e 138 foram exclusivas, sendo 61 para a trilha entre mata ciliar e o talhão de eucalipto e 77 para a trilha entre talhões de eucalipto (Fig. 2).

A análise da abundância evidenciou um número reduzido de espécies com muitos indivíduos e uma elevada proporção de espécies únicas nas duas áreas (Fig. 3). Na trilha entre o remanescente de mata ciliar e o talhão de eucalipto, o modelo de distribuição se ajusta à Série Geométrica ( $\mathrm{p}=0,067)$. MAGURRAN (1988) comenta que este padrão pode ser observado basicamente em estágios temporários de sucessão, situação constatada nos remanescentes de mata ciliar na área de estudo. A

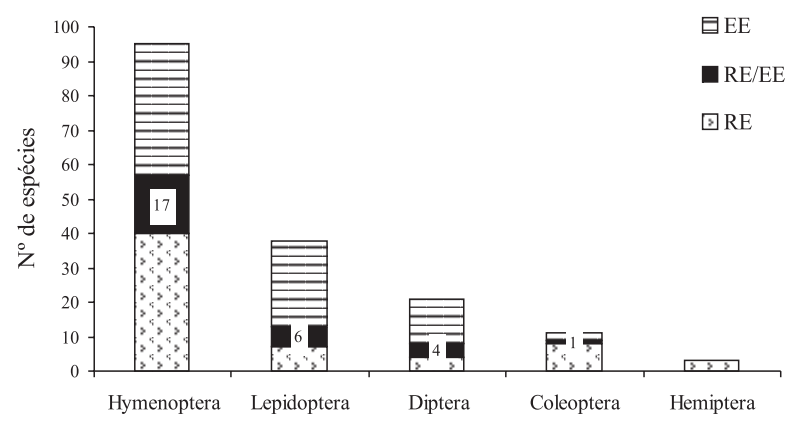

Fig. 2. Representatividade de espécies de insetos antófilos coletados no período de setembro de 2002 a agosto de 2003 , no Horto Florestal AES-SUL, Triunfo, RS. (RE, trilha entre remanescente de mata ciliar e talhão de eucalipto; EE, trilha entre dois talhões de eucalipto; RE/EE, trilha entre remanescente de mata ciliar e talhão de eucalipto e trilha entre dois talhões de eucalipto). 
Tabela I. Insetos antófilos registrados entre setembro de 2002 e agosto de 2003, no Horto Florestal AES-SUL, Triunfo, RS, com o número de indivíduos (NI), número de indivíduos por coleta (nc), meses de ocorrência (MO), constância (C), dominância (D) e o status (A, acessória; Ac, acidental; C, constante; Sd, subdominante; R, recessiva; Rr, rara), respectivamente nas trilhas entre remanescente de mata ciliar e talhão de eucalipto (RE) e entre dois talhões de eucalipto (EE).

\begin{tabular}{lllllllllllll}
\hline & & RE & & & & & & EE & & & & \\
\hline Táxon & NI & nc & MO & C & D & NI & nc & MO & C & D
\end{tabular}

\section{HYMENOPTERA}

APOIDEA (APIFORMES)

Ancylocelis sp.

Anthrenoides sp. nov.

Apis mellifera Linnaeus, 1758

MO C D NI nc

$\mathrm{MO}$

Arhysoceble picta (Friese, 1899)

Augochlora amphitrite

(Schrottky, 1910)

Augochlora tantilla Moure, $1943 \quad 0 \quad 0$

Augochlora sp. 1

Augochlora sp. 2

Augochlora sp. 3

Augochlora sp. 4

Augochlorella michaelis

(Vachal, 1911)

Augochloropsis cupreola

(Cockerell, 1900)

Augochloropsis sparsilis

(Vachal, 1903)

Augochloropsis sp. 1

Augochloropsis sp. 2

Augochloropsis sp. 3

Bombus atratus Franklin, 1913

Cephalurgus anomalus

Moure \& Oliveira, 1962

Ceratina cf. asuncionis

Strand, 1910

Ceratina hyemalis Moure, 1950

Ceratina paraguayensis

Schrottky, 1907

Ceratina sp.

Ceratina (Ceratinula) sp.

Chalepogenus sp.

Coelioxys sp.

Dialictus cf. anisitsianus

(Strand, 1910)

Dialictus sp. 1

Dialictus sp. 2

Dialictus sp. 3

Dialictus sp. 4

Hypanthidium divaricatum

(Smith, 1854)

Megachile sp. 1

Megachile sp. 2

Megachile sp. 3

Megachile sp. 4

Melissodes sp.

Melissoptila bonaerensis

Holmberg, 1903

Melissoptila paraguayensis

Brèthes, 1909

Melissoptila sp. 1

Melissoptila sp. 2

Melitoma segmentaria

$\begin{array}{ccccccc} & 1 & \text { Jan } & \mathrm{Ac} & \mathrm{Rr} & 0 & 0 \\ 2 & 1 & \text { Out } & \mathrm{Ac} & \mathrm{Rr} & 3 & 2 \\ 7 & 4 & \text { Out Mar Ago } & \mathrm{Ac} & \mathrm{Sd} & 11 & 4 \\ 2 & 1 & \text { Fev } & \mathrm{Ac} & \mathrm{Rr} & 0 & 0 \\ 3 & 6 & \text { Dez Fev Mar Mai Jun Jul } & \mathrm{A} & \mathrm{Sd} & 8 & 5\end{array}$

$\begin{array}{ccc}\text { Nov Dez Jan Abr } & \text { A } & \text { Sd } \\ \text { Out Dez Fev Mar Abr Jul } & \text { A } & \text { R } \\ \text { Nov } & \text { Ac } & \mathrm{Rr} \\ \text { Jan } & \text { Ac } & \mathrm{Rr}\end{array}$

Abr
Mar
Nov
Dez

$\begin{array}{llll} & & 2 & 2 \\ & & 1 & 1 \\ \mathrm{Ac} & \mathrm{Rr} & 0 & 0 \\ \mathrm{Ac} & \mathrm{Rr} & 0 & 0 \\ \mathrm{Ac} & \mathrm{Rr} & 0 & 0 \\ \mathrm{Ac} & \mathrm{Rr} & 0 & 0\end{array}$

\begin{tabular}{|c|c|c|c|c|c|c|c|c|}
\hline 1 & 1 & Dez & Ac & $\mathrm{Rr}$ & 6 & 5 & Set Nov Fev Mar Jun & A \\
\hline 3 & 2 & Fev Mar & Ac & $\mathrm{Rr}$ & 0 & 0 & & \\
\hline 0 & 0 & & & & 1 & 1 & Set & Ac \\
\hline 1 & 1 & Fev & Ac & $\mathrm{Rr}$ & 0 & 0 & & \\
\hline 2 & 1 & Ago & Ac & $\mathrm{Rr}$ & 1 & 1 & Mar & Ac \\
\hline 2 & 2 & Out Fev & Ac & $\mathrm{Rr}$ & 0 & 0 & & \\
\hline 0 & 0 & & & & 22 & 7 & Dut Dez Jan Fev Mar Mai & A \\
\hline
\end{tabular}

(Fabricius, 1804)

Odyneropsis sp.

Panurgillus reticulatus

(Schlindwein \& Moure, 1998)

Parapsaenythia sp. nov.

116 Out Nov Jan Fev Mar Mai A $\quad$ Sd $\quad 0 \quad 0$

$\begin{array}{ccccccc}5 & 4 & \text { Out Nov Fev Abr } & \text { A } & \text { Rr } & 0 & 0 \\ 1 & 1 & \text { Mai } & \text { Ac } & \text { Rr } & 0 & 0\end{array}$

0

$0 \quad 0$

82

11

11

Jan Fev
Mar
Fev

Ac $\mathrm{R} \quad 0 \quad 0$

Ac $\operatorname{Rr} \quad 0 \quad 0$

Ac $\operatorname{Rr} 5 \quad 4$

$0 \quad 0$

$0 \quad 0$

$3 \quad 1$

00

32

$0 \quad 0$

22

$0 \quad 0$

11

$\begin{array}{ll}1 & 1 \\ 1 & 1\end{array}$

1

$11 \quad$ Mar

$1 \quad 1 \quad$ Fev

11 Mar

22

Dez Fev

Jan

21

00

$0 \quad 0$

\begin{abstract}
Mar Jun
\end{abstract}
Dez Mar Fev

Nov Jan

Mar

Mar

Mar

Fev

(n)

Dez Fev
Mar Set

Ac $\quad \mathrm{Rr}$

$\begin{array}{llll} & & 1 & 1 \\ & & 1 & 1 \\ \text { Ac } & \operatorname{Rr} & 1 & 1 \\ & & 3 & 1 \\ \text { Ac } & \operatorname{Rr} & 0 & 0\end{array}$

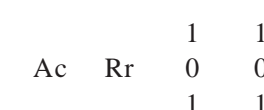

$\begin{array}{lll}\text { Ac } & \operatorname{Rr} & 0 \\ & & \end{array}$

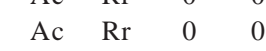$$
\begin{array}{lll}
\text { Ac } & \text { Rr } & 9
\end{array}
$$$$
\text { Ac } \operatorname{Rr} \quad 0 \quad 0
$$$$
\begin{array}{llll}
\text { Ac } & \mathrm{Rr} & 0 & 0
\end{array}
$$$$
\begin{array}{llll}
\text { Ac } & \mathrm{Rr} & 0 & 0
\end{array}
$$$$
\begin{array}{llll}
\text { Ac } & \mathrm{Rr} & 0 & 0 \\
\mathrm{Ac} & \mathrm{Rr} & 0 & 0
\end{array}
$$$$
\text { Ac } \quad \operatorname{Rr} \quad 0 \quad 0
$$ 
$\underline{\text { Tabela I (cont.) }}$

\begin{tabular}{|c|c|c|c|c|c|c|c|c|c|c|}
\hline $\begin{array}{l}\text { Paratetrapedia fervida } \\
\text { (Smith, 1874) }\end{array}$ & 1 & 1 & Jan & Ac & $\mathrm{Rr}$ & 0 & 0 & & & \\
\hline Plebeia droryana (Friese, 1900) & 4 & 2 & Jan Mar & Ac & $\mathrm{Rr}$ & 7 & 1 & Fev & Ac & $\mathrm{R}$ \\
\hline Psaenythia bergi Holmberg, 1884 & 6 & 3 & Nov Dez Jan Fev Ago & Ac & $\mathrm{R}$ & 3 & 2 & Nov Mar & Ac & $\mathrm{Rr}$ \\
\hline Psaenythia litoralis Holmberg, 1923 & 4 & 4 & Nov Dez Jan Fev Ago & Ac & $\mathrm{Rr}$ & 2 & 2 & Nov Mar & Ac & $\mathrm{Rr}$ \\
\hline $\begin{array}{l}\text { Pseudagapostemon pruinosus } \\
\text { Moure \& Sakagami, } 1984\end{array}$ & 2 & 2 & Dez Abr & Ac & $\mathrm{Rr}$ & 1 & 1 & Fev & Ac & $\mathrm{Rr}$ \\
\hline $\begin{array}{l}\text { Pseudauglochlora graminea } \\
\text { (Fabricius, 1804) }\end{array}$ & 0 & 0 & & & & 1 & 1 & Fev & Ac & $\mathrm{Rr}$ \\
\hline Ptilothrix relata (Holmberg, 1903) & 2 & 1 & Dez & Ac & $\mathrm{Rr}$ & 2 & 2 & Fev Dez & Ac & $\mathrm{Rr}$ \\
\hline Ptilothrix sp. & 1 & 1 & Nov & Ac & $\mathrm{Rr}$ & & & & & \\
\hline Xylocopa frontalis (Olivier, 1789) & 1 & 1 & Dez & Ac & $\mathrm{Rr}$ & 0 & 0 & & & \\
\hline \multicolumn{11}{|l|}{ APOIDEA (SPHECIFORMES) } \\
\hline $\begin{array}{l}\text { Alphamenes campanulatus } \\
\text { (Fabricius, 1804) }\end{array}$ & 1 & 1 & Ago & Ac & $\mathrm{Rr}$ & 0 & 0 & & & \\
\hline Ancistroceroides sp. & 0 & 0 & & & & 1 & 1 & Nov & Ac & $\mathrm{Rr}$ \\
\hline Ancistocerus sp. & 2 & 2 & Out Nov & Ac & $\mathrm{Rr}$ & 1 & 1 & Nov & Ac & $\operatorname{Rr}$ \\
\hline Anoplius inaurata (Smith, 1879) & 0 & 0 & & & & 3 & 1 & Nov & Ac & $\mathrm{Rr}$ \\
\hline $\begin{array}{l}\text { Brachygastra lecheguana } \\
\text { (Latreille, 1824) }\end{array}$ & 4 & 2 & Nov & Ac & $\mathrm{Rr}$ & 0 & 0 & & & \\
\hline Cerceris sp. & 0 & 0 & & & & 1 & 1 & Nov & Ac & $\mathrm{Rr}$ \\
\hline $\begin{array}{l}\text { Chlorion hemipyrrhum } \\
\text { (Sichel, 1863) }\end{array}$ & 1 & 1 & Mar & Ac & $\mathrm{Rr}$ & 0 & 0 & & & \\
\hline Chrysis sp. & 0 & 0 & & & & 1 & 1 & Nov & $\mathrm{Ac}$ & $\mathrm{Rr}$ \\
\hline $\begin{array}{l}\text { Entypus ferruginipennis } \\
\text { (Haliday, 1837) }\end{array}$ & 0 & 0 & & & & 8 & 7 & Jan Mar Nov & Ac & $\mathrm{R}$ \\
\hline Hypancistrocerus sp. & 0 & 0 & & & & 2 & 2 & Dez Mar & Ac & $\mathrm{Rr}$ \\
\hline Hypalastoroides sp. & 2 & 2 & Out Nov Fev & Ac & $\mathrm{Rr}$ & 1 & 1 & Mai Nov & $\mathrm{Ac}$ & $\mathrm{Rr}$ \\
\hline Idionysson sp. & 0 & 0 & & & & 1 & 1 & Dez & Ac & $\mathrm{Rr}$ \\
\hline $\begin{array}{l}\text { Mischocyttarus drewseni } \\
\text { Saussure, } 1857\end{array}$ & 1 & 1 & Ago & Ac & $\mathrm{Rr}$ & 0 & 0 & & & \\
\hline $\begin{array}{l}\text { Montezumia nigriceps } \\
\quad \text { (Spinola, 1841) }\end{array}$ & 0 & 0 & & & & 1 & 1 & Nov & Ac & $\mathrm{Rr}$ \\
\hline Stenodynerus sp. & 1 & 1 & Nov & Ac & $\mathrm{Rr}$ & 6 & 2 & Fev Mar & Ac & $\mathrm{R}$ \\
\hline $\begin{array}{l}\text { Stenonartonia guaranitica } \\
\text { (Bertoni, 1918) }\end{array}$ & 1 & 1 & Mai & Ac & $\mathrm{Rr}$ & 0 & 0 & & & \\
\hline $\begin{array}{l}\text { Omicron aurantiopictum } \\
\text { Soika, } 1978\end{array}$ & 1 & 1 & Ago & Ac & $\mathrm{Rr}$ & 0 & 0 & & & \\
\hline $\begin{array}{l}\text { Podagritus bocainus } \\
\text { Leclereq, } 1982\end{array}$ & 0 & 0 & & & & 1 & 1 & Out & Ac & $\mathrm{Rr}$ \\
\hline Polistes actaeon Haliday, 1836 & 2 & 2 & Mar Abr & Ac & $\mathrm{Rr}$ & 0 & 0 & & & \\
\hline Polistes cinerascens Saussure, 1854 & 6 & 3 & Jan Mar Ago & Ac & $\mathrm{R}$ & 0 & 0 & & & \\
\hline Polybia ignobilis (Haliday, 1836) & 0 & 0 & & & & 1 & 1 & Nov & $\mathrm{Ac}$ & $\mathrm{Rr}$ \\
\hline Polybia sericea (Olivier, 1791) & 0 & 0 & & & & 1 & 1 & Nov & Ac & $\mathrm{Rr}$ \\
\hline Prionyx thomae (Fabricius. 1775) & 0 & 0 & & & & 7 & 4 & Nov Jan Fev Mar & Ac & $\mathrm{R}$ \\
\hline \multicolumn{11}{|l|}{ LEPIDOPTERA } \\
\hline Achlyodes mithradates (Jung, 1792) & 0 & 0 & & & & 2 & 1 & Nov & Ac & $\mathrm{Rr}$ \\
\hline Actinote sp. & 1 & 1 & Mar & Ac & $\mathrm{Rr}$ & 0 & 0 & & & \\
\hline Adelpha syma (Godart, 1824) & 1 & 1 & Mai & Ac & $\mathrm{Rr}$ & 4 & 2 & Mai Ago & $\mathrm{Ac}$ & $\mathrm{Rr}$ \\
\hline $\begin{array}{l}\text { Agraulis vanillae maculosa } \\
\text { (Stichel, 1907) }\end{array}$ & 0 & 0 & & & & 7 & 3 & Jan Fev Dez & Ac & $\mathrm{R}$ \\
\hline $\begin{array}{l}\text { Anartia amathea roeselia } \\
\text { (Eschscholtz, 1821) }\end{array}$ & 6 & 3 & Mar Jan & Ac & $\mathrm{R}$ & 2 & 1 & Jan & Ac & $\mathrm{Rr}$ \\
\hline Arawacus separata (Lathy, 1926) & 0 & 0 & & & & 1 & 1 & Fev & Ac & $\mathrm{Rr}$ \\
\hline Callimormus beda (Plötz, 1886) & 0 & 0 & & & & 7 & 3 & Nov Mar Dez & Ac & $\mathrm{R}$ \\
\hline $\begin{array}{l}\text { Dryadula phaetusa } \\
\text { (Linnaeus, 1758) }\end{array}$ & 0 & 0 & & & & 1 & 1 & Mai & Ac & $\mathrm{Rr}$ \\
\hline Dryas iulia (Fabricius, 1775) & 3 & 2 & Mai Mar & Ac & $\mathrm{Rr}$ & 7 & 6 & Ago Dez Mai Jan Fev Nov & A & $\mathrm{R}$ \\
\hline Gorgythion b. begga (Kirby, 1871) & 0 & 0 & & & & 2 & 2 & Nov Dez & Ac & $\mathrm{Rr}$ \\
\hline $\begin{array}{l}\text { Helias phalaenoides palpalis } \\
\text { (Latreille, 1824) }\end{array}$ & 1 & 1 & Jan & Ac & $\mathrm{Rr}$ & 0 & 0 & & & \\
\hline $\begin{array}{l}\text { Heliconius erato phyllis } \\
\text { (Fabricius, 1775) }\end{array}$ & 2 & 2 & Mar Mai & Ac & $\mathrm{Rr}$ & 22 & & go Nov Dez Jan Fev Mar N & Iai C & $\mathrm{Sd}$ \\
\hline Heliopetes alana Reakirt, 1868 & 0 & 0 & & & & 1 & 1 & Mar & Ac & $\mathrm{Rr}$ \\
\hline Heliopetes omrina (Butler, 1870) & 0 & 0 & & & & 1 & 1 & Fev & Ac & $\mathrm{Rr}$ \\
\hline
\end{tabular}


Tabela I (cont.)

\begin{tabular}{|c|c|c|c|c|c|c|c|c|c|c|}
\hline $\begin{array}{l}\text { Hermeuptychia hermes } \\
\text { (Fabricius, 1775) }\end{array}$ & 0 & 0 & & & & 1 & 1 & Set & Ac & $\mathrm{Rr}$ \\
\hline Mechanitis lysimnia (Fabricius, 1793) & 0 & 0 & & & & 1 & 1 & Mai & Ac & $\mathrm{Rr}$ \\
\hline Pyrginae sp. 1 & 1 & 1 & Jan & A & $\mathrm{Rr}$ & 0 & 0 & & & \\
\hline Pyrginae sp. 2 & 0 & 0 & & & & 2 & 2 & Jul Jan & Ac & $\mathrm{Rr}$ \\
\hline Pyrginae sp. 3 & 1 & 1 & Abr & Ac & $\mathrm{Rr}$ & 3 & 3 & Set Mar Dez & Ac & $\mathrm{Rr}$ \\
\hline Pyrginae sp. 4 & 0 & 0 & & & & 2 & 2 & Jun Set & $\mathrm{Ac}$ & $\mathrm{Rr}$ \\
\hline Pyrginae sp. 5 & 0 & 0 & & & & 1 & 1 & $\mathrm{Fev}$ & Ac & $\mathrm{Rr}$ \\
\hline Pyrginae sp. 6 & 1 & 1 & Dez & Ac & $\mathrm{Rr}$ & 1 & 1 & Fev & Ac & $\mathrm{Rr}$ \\
\hline Pyrginae sp. 7 & 0 & 0 & & & & 1 & 1 & Set & Ac & $\mathrm{Rr}$ \\
\hline Pyrginae sp. 8 & 0 & 0 & & & & 1 & 1 & Set & $\mathrm{Ac}$ & $\mathrm{Rr}$ \\
\hline Pyrginae sp. 9 & 0 & 0 & & & & 1 & 1 & Mar & Ac & $\mathrm{Rr}$ \\
\hline Pyrginae sp. 10 & 0 & 0 & & & & 1 & 1 & Dez & Ac & $\mathrm{Rr}$ \\
\hline Pyrginae sp. 11 & 0 & 0 & & & & 4 & 3 & Set Jan Jul & Ac & $\mathrm{Rr}$ \\
\hline Pyrginae sp. 12 & 0 & 0 & & & & 4 & 3 & Fev Jun Jul & Ac & $\mathrm{Rr}$ \\
\hline Pyrginae sp. 13 & 1 & 1 & Mar & Ac & $\mathrm{Rr}$ & 0 & 0 & & & \\
\hline Pyrginae sp. 14 & 0 & 0 & & & & 2 & 2 & Jul Set & Ac & $\mathrm{Rr}$ \\
\hline Polites vibex catilina (Plötz, 1886) & 1 & 1 & Jan & Ac & $\mathrm{Rr}$ & 1 & 1 & Jan & Ac & $\mathrm{Rr}$ \\
\hline Pyrgus orcus (Stoll, 1780) & 0 & 0 & & & & 20 & 6 & Nov Dez Jan Mar Mai Ago & A & $\mathrm{Sd}$ \\
\hline $\begin{array}{l}\text { Tegosa claudina } \\
\text { (Eschscholtz, 1821) }\end{array}$ & 4 & 2 & Nov Dez & Ac & $\mathrm{Rr}$ & 0 & 0 & & & \\
\hline $\begin{array}{l}\text { Trina g. geometrina } \\
\text { (Felder \& Felder, 1867) }\end{array}$ & 0 & 0 & & & & 4 & 3 & Nov Mai Jun & $\mathrm{Ac}$ & $\mathrm{Rr}$ \\
\hline Urbanus teleus (Hübner, 1821) & 0 & 0 & & & & 2 & 2 & Dez Mai & Ac & $\mathrm{Rr}$ \\
\hline Vehilius s. stictomenes (Butler, 1877) & 0 & 0 & & & & 1 & 1 & Mar & Ac & $\mathrm{Rr}$ \\
\hline Zariaspes mys (Hübner, 1808) & 1 & 1 & Dez & Ac & $\mathrm{Rr}$ & 0 & 0 & & & \\
\hline \multicolumn{11}{|l|}{ IPTERA } \\
\hline Asilidae sp. & 1 & 1 & Fev & Ac & $\mathrm{Rr}$ & 0 & 0 & & & \\
\hline Bibionidae sp. & 0 & 0 & & & & 3 & 2 & Out Mar & Ac & $\mathrm{Rr}$ \\
\hline Bombyliidae sp. 1 & 0 & 0 & & & & 1 & 1 & Nov & Ac & $\mathrm{Rr}$ \\
\hline Bombyliidae sp. 2 & 0 & 0 & & & & 1 & 1 & Jan & Ac & $\mathrm{Rr}$ \\
\hline Diptera sp. 1 & 2 & 1 & Out & $\mathrm{Ac}$ & $\mathrm{Rr}$ & 0 & 0 & & & \\
\hline Diptera sp. 2 & 1 & 1 & Set & Ac & $\mathrm{Rr}$ & 0 & 0 & & & \\
\hline Diptera sp. 3 & 0 & 0 & & & & 1 & 1 & Out & $\mathrm{Ac}$ & $\mathrm{Rr}$ \\
\hline Diptera sp. 4 & 0 & 0 & & & & 1 & 1 & Out & $\mathrm{Ac}$ & $\mathrm{Rr}$ \\
\hline Diptera sp. 5 & 0 & 0 & & & & 1 & 1 & Mar & Ac & $\mathrm{Rr}$ \\
\hline Dolichopodidae sp. & 0 & 0 & & & & 1 & 1 & Dez & $\mathrm{Ac}$ & $\mathrm{Rr}$ \\
\hline Eristalis sp. 1 & 1 & 1 & Set & Ac & $\mathrm{Rr}$ & 0 & 0 & & & \\
\hline Eristalis sp. 2 & 3 & 2 & Set Nov & $\mathrm{Ac}$ & $\mathrm{Rr}$ & 3 & 2 & Nov Dez & Ac & $\mathrm{Rr}$ \\
\hline Eristalis sp. 3 & 0 & 0 & & & & 2 & 1 & Nov & Ac & $\mathrm{Rr}$ \\
\hline Masarygus sp. & 1 & 1 & Jan & Ac & $\mathrm{Rr}$ & 2 & 2 & Jan & Ac & $\mathrm{Rr}$ \\
\hline Otitidae sp. & 0 & 0 & & & & 3 & 2 & Jan Mar & Ac & $\mathrm{Rr}$ \\
\hline Salpingogaster sp. & 1 & 1 & Dez & Ac & $\mathrm{Rr}$ & 8 & 4 & Dez Jan Fev Mai & A & $\mathrm{R}$ \\
\hline Sarcophagidae sp. & 0 & 0 & & & & 3 & 2 & Set Nov & Ac & $\mathrm{Rr}$ \\
\hline Syrphidae sp. & 0 & 0 & & & & 1 & 1 & Mai & $\mathrm{Ac}$ & $\mathrm{Rr}$ \\
\hline Tachinidae sp. & 0 & 0 & & & & 1 & 1 & Nov & Ac & $\mathrm{Rr}$ \\
\hline Toxomerus sp. 1 & 2 & 2 & Set Out & $\mathrm{Ac}$ & $\mathrm{Rr}$ & 10 & 1 & Nov & Ac & $\mathrm{R}$ \\
\hline Toxomerus sp. 2 & 1 & 1 & Set & Ac & $\mathrm{Rr}$ & 9 & 3 & Nov Dez & Ac & $\mathrm{R}$ \\
\hline \multicolumn{11}{|l|}{ OLEOPTERA } \\
\hline Buprestidae sp. & 2 & 2 & Nov & Ac & $\mathrm{Rr}$ & 0 & 0 & & & \\
\hline Chrysomelidae sp. & 1 & 1 & Dez & Ac & $\mathrm{Rr}$ & 0 & 0 & & & \\
\hline Curculionidae sp. & 1 & 1 & Jan & Ac & $\mathrm{Rr}$ & 0 & 0 & & & \\
\hline Euphoria lurida (Fabricius, 1775) & 1 & 1 & Mar & Ac & $\mathrm{Rr}$ & 1 & 1 & Mar & Ac & $\mathrm{Rr}$ \\
\hline Macraspis sp. & 5 & 1 & Dez & Ac & $\mathrm{Rr}$ & 0 & 0 & & & \\
\hline Megacerus reticulatus (Sharp, 1885) & 1 & 1 & Jul & Ac & $\mathrm{Rr}$ & 0 & 0 & & & \\
\hline Meloidae sp. & 2 & 1 & Fev & Ac & $\mathrm{Rr}$ & 0 & 0 & & & \\
\hline Rutela lineola (Linnaeus, 1767) & 2 & 1 & Dez & Ac & $\mathrm{Rr}$ & 0 & 0 & & & \\
\hline Tenebrionidae sp. & 0 & 0 & & & & 1 & 1 & Jan & Ac & $\mathrm{Rr}$ \\
\hline Trichaltica sp. & 0 & 0 & & & & 1 & 1 & Dez & Ac & $\mathrm{Rr}$ \\
\hline $\begin{array}{l}\text { Zonitolytta chrysomeloides } \\
\text { (Linnaeus, 1763) }\end{array}$ & 7 & 2 & Dez Jan & Ac & $\mathrm{R}$ & 0 & 0 & & & \\
\hline \multicolumn{11}{|l|}{ EMIPTERA } \\
\hline Pentatomidae sp. & 1 & 1 & Nov & Ac & $\mathrm{Rr}$ & 0 & 0 & & & \\
\hline Lygaeidae sp. 1 & 3 & 2 & Mar Ago & $\mathrm{Ac}$ & $\mathrm{Rr}$ & 0 & 0 & & & \\
\hline Lygaeidae sp. 2 & 1 & 1 & Set & Ac & $\mathrm{Rr}$ & 0 & 0 & & & \\
\hline
\end{tabular}


mesma autora infere que o modelo geométrico também se aplica em ambientes pobres quanto ao número de espécies, sendo esta uma característica observada na trilha com remanescente de mata ciliar do presente estudo, relativamente à outra área. A trilha entre os dois talhões de eucalipto está caracterizada pelo modelo Log-normal ( $\mathrm{p}=0,041)$ de distribuição, assinalando comunidades que sofrem a influência de numerosos fatores, resultando em diferentes processos que determinaram este padrão de distribuição (MAGURRAN, 1988).

Nas trilhas com mata ciliar e eucalipto e entre talhões de eucalipto, respectivamente $95,6 \%$ e $91,5 \%$ das espécies amostradas $(n=90$ e $n=106)$ foram consideradas acidentais e 4,4\% e 7,5\% acessórias. Nenhuma espécie foi constante na trilha entre remanescente de mata ciliar e talhão de eucalipto e apenas Heliconius erato phyllis (Fabricius, 1775) foi constante entre os dois talhões de eucalipto (Tab. I). Amostras maiores provavelmente possibilitariam melhor representatividade das espécies de visitantes florais, embora espécies sazonais apresentem atividades restritas a curtos períodos de tempo, a exemplo de himenópteros solitários. O período de maior ocorrência de insetos foi de novembro de 2002 a março de 2003 (Fig. 4), quando as temperaturas foram mais elevadas, havendo uma correlação altamente significativa em relação à temperatura, tanto na trilha entre remanescente de mata ciliar e talhão de eucalipto $(\mathrm{r}=$ $0,765, \mathrm{p}=0,004)$ como entre os dois talhões de eucalipto $(\mathrm{r}=0,790, \mathrm{p}=0,002)$. No caso da precipitação, não houve correlação significativa.

Associada à baixa constância da maioria das espécies, observou-se também uma dominância reduzida das mesmas. Dentre as espécies amostradas nas duas áreas, nenhuma foi dominante ou eudominante. As espécies que obtiveram ocorrência superior a $2 \%$ (subdominantes) foram Apis mellifera Linnaeus, 1758, Augochlora amphitrite (Schrottky, 1910) e Ceratina cf. asuncionis Strand, 1910 na trilha entre remanescente de mata ciliar e talhão de eucalipto e Apis mellifera, Cephalurgus anomalus Moure \& Oliveira, 1962, Heliconius erato phyllis e Pyrgus orcus (Stoll, 1780), na trilha entre os dois talhões de eucalipto. As demais espécies foram recessivas ou raras (Tab. I).

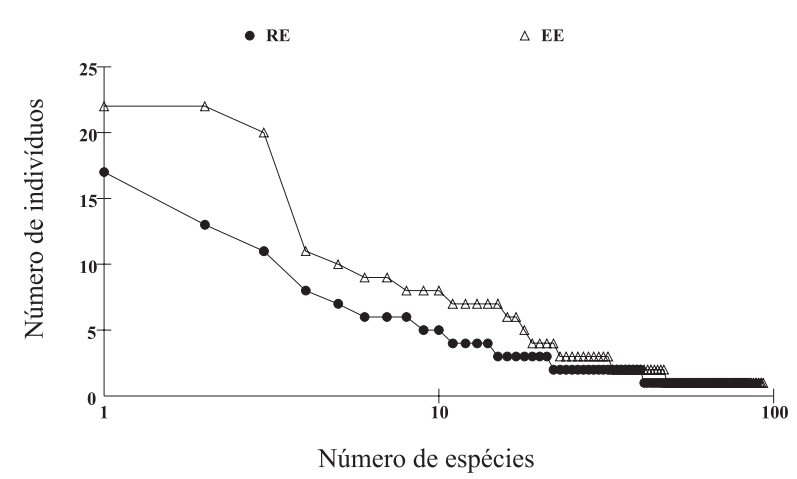

Fig. 3. Curva de distribuição da abundância das espécies de insetos visitantes florais coletadas no período de setembro de 2002 a agosto de 2003, no Horto Florestal AES-SUL, Triunfo, RS. (RE, trilha entre remanescente de mata ciliar e talhão de eucalipto; EE, trilha entre dois talhões de eucalipto).
Em consonância com os resultados obtidos no presente estudo, Hingston \& PotTs (1998) verificaram, em uma comunidade de visitantes florais na Tasmânia, que a introduzida Apis mellifera foi a espécie dominante. Espécies generalistas são freqüentemente capazes de usar diferentes tipos de habitat ou distintas porções de um habitat (borda e interior) (GOLDEN \& CRIST, 1999). WiLMs et al. (1996) e AGUIAR \& MARTINS (2003) observaram que Apis mellifera representou mais que $20 \%$ dos espécimes coletados, sugerindo o possível efeito negativo na comunidade de abelhas como potencial competidor exótico. Brown JR. \& FreITAS (2002) apontam que a composição típica da fauna em cada região parece responder diferencialmente à área, distúrbios e vários fatores micro-ambientais, em parte devido às diversas espécies comuns ou dominantes e à presença de distintos recursos das plantas.

Diversidade. A análise dos grupos de insetos antófilos no Horto Florestal AES-SUL, demonstrada pelos índices de Shannon-Wiener e Simpson, revelou alta diversidade de espécies e valores semelhantes para as duas áreas de estudo (Tab. II). Segundo MagurRan (1988), o índice de Shannon-Wiener expressa a uniformidade dos valores através de todas as amostras e raramente ultrapassa 4,5. O índice de Simpson, que é influenciado pela importância das espécies mais dominantes, também evidenciou semelhança na heterogeneidade da fauna nas duas áreas. Entretanto, em relação à equitabilidade (Pielou), a distribuição de espécies na trilha entre remanescente de mata ciliar e talhão de eucalipto apresentou-se mais equilibrada (Tab. II).

Considerando-se o alto grau de antropização verificado no horto, seriam esperadas comunidades de insetos antófilos com baixa diversidade. Entretanto, contrariamente verificou-se alta diversidade desta fauna nas duas áreas estudadas. Este resultado pode estar relacionado à capacidade de deslocamento dos grupos de insetos, a partir de refúgios estabelecidos em pequenas parcelas com ambientes favoráveis mantidos na região. Poggiani \& Ollveira (1998) teorizam que a diversidade e abundância da fauna e flora são determinadas pela amplitude de nichos adequados à sua sobrevivência.

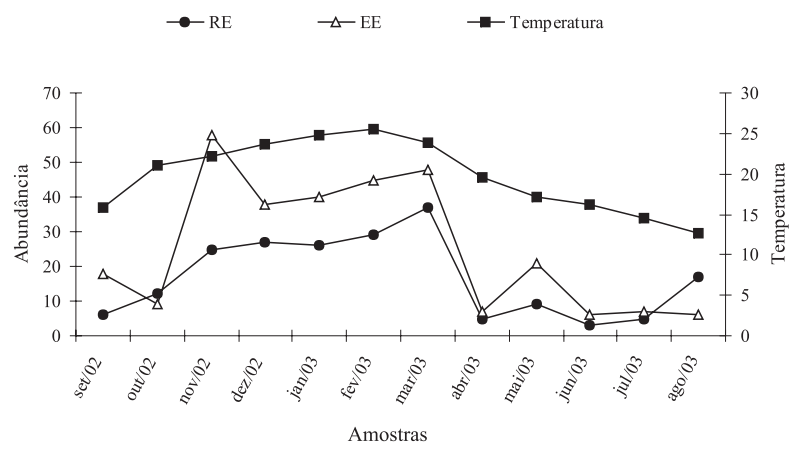

Fig. 4. Número de insetos visitantes florais coletados e médias de temperatura registradas no período de setembro de 2002 a agosto de 2003, no Horto Florestal AES-SUL, Triunfo, RS. (RE, trilha entre remanescente de mata ciliar e talhão de eucalipto; EE, trilha entre dois talhões de eucalipto). 
Ambientes que são perturbados periodicamente tendem a apresentar diversidade mais alta do que ecossistemas em "equilíbrio" (Huston, 1979). Alguns autores destacam que o efeito de borda pode aumentar a diversidade de espécies (DENYs \& TSCHARNTKE, 2002; Tscharntke et al., 2002; TscharntKe \& BRANDL, 2004). Provavelmente a alta diversidade de insetos visitantes florais observada na área estudada é decorrente do efeito de borda causado pelos talhões de eucalipto.

O número esperado de espécies, baseado nas curvas de rarefação, indica que a diversidade de insetos florais foi distinta nas duas áreas amostradas, considerando-se uma amostragem de 201 indivíduos. As curvas de rarefação não alcançaram a assimptota, indicando que o número máximo de espécies esperadas nas duas áreas não foi atingido (Fig. 5).

$\mathrm{O}$ coeficiente de similaridade de Sorensen $(\mathrm{S}=$ 0,239 ) indica que as áreas amostradas são distintas quanto à composição de espécies de insetos, levando em consideração a presença ou ausência destas (Tab. I). Analisando-se a comunidade de insetos antófilos no Horto Florestal AES-Sul conclui-se que esta diferiu entre as áreas quanto ao número de indivíduos e riqueza de espécies. Entretanto não houve diferença significativa quanto sua estrutura, revelada pelos resultados dos índices de diversidade aplicados (Tab. II).

Insetos antófilos predominantes. Dentre os grupos de insetos coletados no horto, percebe-se maior diversidade entre os himenópteros - especialmente entre

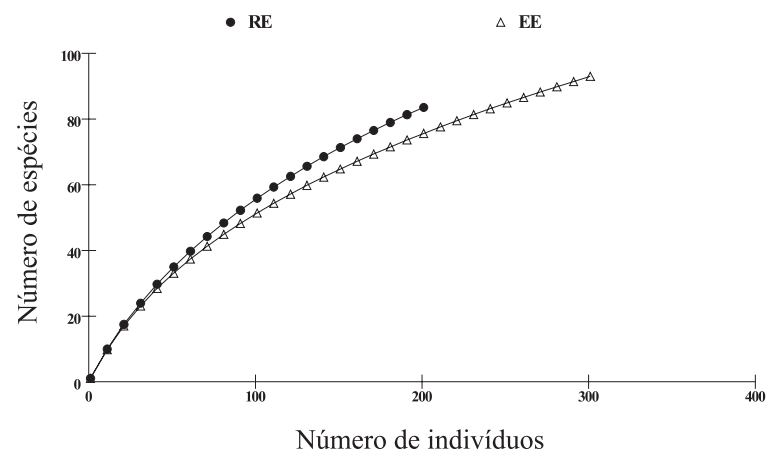

Fig. 5. Curvas de Rarefação das comunidades dos insetos visitantes florais coletados no período de setembro de 2002 a agosto de 2003, no Horto Florestal AES-SUL, Triunfo, RS. (RE, trilha entre remanescente de mata ciliar e talhão de eucalipto; EE, trilha entre dois talhões de eucalipto).

Tabela II. Número de espécies, indivíduos e índices de diversidade relativos aos insetos antófilos coletados no período de setembro de 2002 a agosto de 2003, no Horto Florestal AES-SUL, Triunfo, RS. (RE, trilha entre remanescente de mata ciliar e talhão de eucalipto; $\mathrm{EE}$, trilha entre dois talhões de eucalipto).

\begin{tabular}{lrr}
\hline & \multicolumn{1}{c}{ RE } & \multicolumn{1}{c}{ EE } \\
\hline Espécies & 84 & 93 \\
Indivíduos & 203 & 301 \\
Shannon & 4,084 & 4,018 \\
Simpson & 0,9761 & 0,9719 \\
Equitabilidade Pielou & 0,9217 & 0,8865
\end{tabular}

as abelhas - seguida dos lepidópteros; estas duas ordens representam insetos visitantes florais com importante função no habitat, como potenciais polinizadores. PASCARELla et al. (2001), ao estudarem insetos visitantes florais, enfatizam que algumas espécies podem não ser efetivas polinizadoras e outras apenas se alimentam de estruturas florais ou sementes (dípteros e coleópteros), usam as flores como locais para capturar presas (coleópteros e vespas) ou como locais de repouso (dípteros).

Abelhas. A distribuição relativa das 218 abelhas obtidas neste estudo, das famílias Apidae (44\%), Halictidae (29\%), Andrenidae (23\%) e Megachilidae (4\%), assemelha-se à registrada no nordeste do Rio Grande do Sul (Wilms et al., 1997; Harter, 1999). Apenas Colletidae não foi amostrada no Horto Florestal AES-SUL, família que possui baixa representatividade em outros estudos no Brasil (WitTMAnN \& HoffMAnN, 1990; CuRE et al., 1992; Barbola \& Laroca, 1993; Antonini \& Martins, 2003).

O local onde ocorreu maior abundância e riqueza de espécies de abelhas foi na trilha entre remanescente de mata ciliar e talhão de eucalipto (Tab. III), a qual apresentou modelo de distribuição de abundância do tipo Série Geométrica $(\mathrm{p}=0,057)$, que caracteriza áreas em sucessão (Fig. 6). De acordo com AnTONini \& MARTins (2003), uma variabilidade em componentes no habitat é importante para abelhas dentro de áreas limitadas, tais como fontes de pólen e néctar disponíveis em flores de ervas daninhas e locais para nidificação. Provavelmente

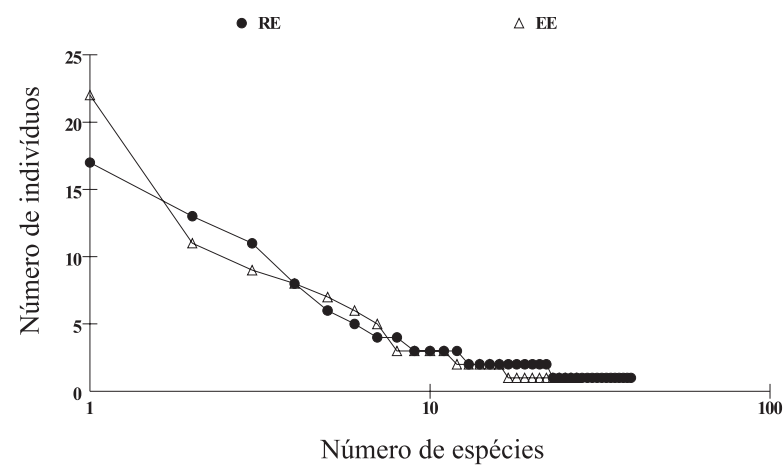

Fig. 6. Curva de distribuição de abundância das espécies de abelhas coletadas no período de setembro de 2002 a agosto de 2003, no Horto Florestal AES-SUL, Triunfo, RS. (RE, trilha entre remanescente de mata ciliar e talhão de eucalipto; EE, trilha entre dois talhões de eucalipto).

Tabela III. Número de espécies, indivíduos e índices de diversidade relativos às abelhas coletadas no período de setembro de 2002 a agosto de 2003, no Horto Florestal AES-SUL, Triunfo, RS. (RE, trilha entre remanescente de mata ciliar e talhão de eucalipto; EE, trilha entre dois talhões de eucalipto).

\begin{tabular}{lrr}
\hline & \multicolumn{1}{c}{ RE } & \multicolumn{1}{c}{ EE } \\
\hline Espécies & 39 & 27 \\
Indivíduos & 117 & 101 \\
Shannon & 3,211 & 2,8 \\
Simpson & 0,9394 & 0,9091 \\
Equitabilidade Pielou & 0,8765 & 0,8496 \\
\hline
\end{tabular}


a maior abundância de abelhas - verificada na trilha entre remanescente de mata ciliar e talhão de eucalipto - está relacionada à ocorrência de estágios sucessionais da vegetação. Aguiar \& MARTins (2003), em dados obtidos na Reserva Biológica de Guaribas, na Paraíba, verificaram uma distribuição do tipo Série Geométrica, com muitas espécies com número reduzido de indivíduos (acima de $50 \%$ das espécies com menos que sete indivíduos) e poucas espécies com muitos indivíduos. No presente estudo, foram verificadas $85,9 \%$ das espécies contendo menos de sete indivíduos; este padrão mostra uma tendência à presença de espécies dominantes na ocupação de sucessivos nichos.

Na trilha entre dois talhões de eucalipto, o padrão observado segue o modelo Log-normal ( $\mathrm{p}=0,071)$ (Fig. 6), semelhante àquele verificado na comunidade de antófilos na mesma área. Este resultado corrobora o padrão encontrado por TAURA \& LAROCA (2001) em área urbana do Paraná.

As 61 espécies de abelhas verificadas nas duas áreas de estudo pertencem à Apidae (48\%), Halictidae (27\%), Andrenidae (16\%) e Megachilidae (9\%). Os índices de diversidade de abelhas em Triunfo, RS, foram altos (Tab. III), quando comparados com os valores apontados por MAGURRAN (1988).

Na trilha entre remanescente de mata ciliar e talhão de eucalipto houve maior diversidade de abelhas, semelhante ao índice registrado $\left(\mathrm{H}^{\prime}=3,374\right)$ em uma reserva biológica na Paraíba por AGUIAR \& MARTINs (2003). $\mathrm{Na}$ trilha entre dois talhões de eucalipto, a diversidade de abelhas foi menor e assemelha-se ao valor encontrado em ambiente antropizado no Paraná (H' = 2,9930) (TAURA \& LAROCA, 2001).

A similaridade entre as duas áreas, de acordo com o índice de Sorensen $(0,32)$, foi distinta quanto à presença de espécies de abelhas. As espécies comuns às duas áreas foram Apis mellifera, Augochlora amphitrite, Augochloropsis cupreola (Cockerell, 1900), Augochloropsis sp. 3, Dialictus cf. anisitrianus (Strand, 1910), Dialictus sp. 3, Melissoptila bonaerensis Holmberg, 1903, Plebeia droryana (Friese, 1900), Psaenythia bergi Holberg, 1884, P. litoralis Holberg, 1923, Pseudagapostemom pruinosus Moure \& Sakagami, 1984 e Ptilothix relata (Holberg, 1903).

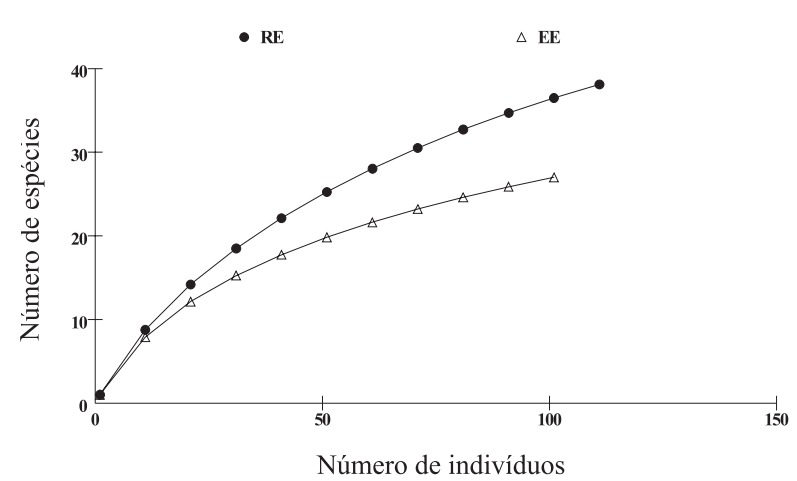

Fig. 7. Curvas de Rarefação da comunidade de abelhas coletadas no período de setembro de 2002 a agosto de 2003, no Horto Florestal AES-SUL, Triunfo, RS. (RE, trilha entre remanescente de mata ciliar e talhão de eucalipto; EE, trilha entre dois talhões de eucalipto).
Plebeia droryana foi a única espécie de meliponíneo registrada no horto florestal. A ausência de outras de abelhas sociais nativas sinaliza a carência de locais para a nidificação (ocos de árvores) e a descontinuidade de oferta de recursos florais ao longo do ano.

As curvas de rarefação para as espécies de abelhas nas duas áreas refletem o padrão demonstrado para a comunidade antófila contemplada no Horto Florestal AESSUL, não tendo atingido a assimptota (Fig. 7). Situação semelhante foi observada por AgUIAR \& MARTINs (2003), que sugerem a necessidade de maior esforço amostral para coletar as espécies raras.

Considerando-se a comunidade de abelhas na área estudada, conclui-se que a maior diversidade (riqueza e estrutura) e número de indivíduos foram registrados na trilha entre remanescente de mata ciliar e talhão de eucalipto, comparativamente à trilha entre os dois talhões de eucalipto.

Plantas visitadas. Os insetos visitaram flores de 31 espécies vegetais de arbustos, arvoretas, lianas e ervas, distribuídas em 20 famílias (Tab. IV). Dentre estas, as que apresentaram maior número de espécies de insetos visitantes florais foram Asteraceae, Malvaceae, Rubiaceae e Onagraceae (Fig. 8). Representantes destas famílias são comumente encontradas em áreas de transição. A vegetação rasteira pode ser caracterizada tipicamente como pioneira em estágios de sucessão florestal. Contudo, uma proporção importante de espécies ruderais pode ser indicativa da fragilidade da comunidade de plantas (HutTel \& Loumeto, 2001).

$\mathrm{Na}$ trilha entre remanescente de mata ciliar e talhão de eucalipto, 24 espécies de plantas foram registradas enquanto que na trilha entre os dois talhões de eucalipto 16; nove espécies vegetais foram comuns às duas áreas. A planta mais visitada foi Sida carpinifolia (Malvaceae), de ocorrência exclusiva da trilha entre os dois talhões de eucalipto; espécies herbáceas da família Malvaceae são pioneiras e invasoras, características que possibilitam a rápida colonização de ambientes ruderais (GOTTSBERGER, 1986). Segundo GaGLiAnONE (2000), a grande propagação destas plantas é devida à independência de polinizadores específicos para sua reprodução, conforme se observou no horto florestal, onde 41 espécies de insetos visitaram S. carpinifolia.

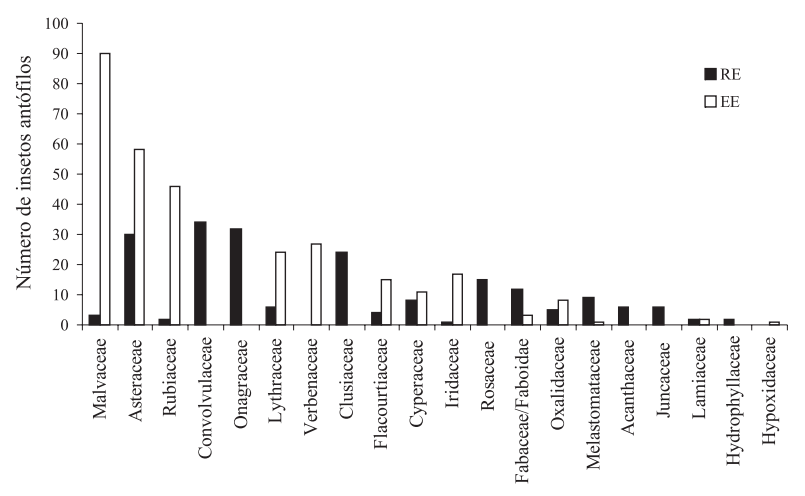

Fig. 8. Famílias de plantas visitadas por insetos antófilos no período de setembro de 2002 a agosto de 2003, no Horto Florestal AES-SUL, Triunfo, RS. (RE, trilha entre remanescente de mata ciliar e talhão de eucalipto; EE, trilha entre dois talhões de eucalipto). 
Tabela IV. Plantas e seus respectivos insetos visitantes florais registrados entre setembro de 2002 e agosto de 2003 , no Horto Florestal AES-SUL, Triunfo, RS. (RE, trilha entre remanescente de mata ciliar e talhão de eucalipto; EE, trilha entre dois talhões de eucalipto).

\section{Plantas}

Sida carpinifolia

Pavonia hastata

ASTERACEAE

Elephantopus mollis

Mikania orleansensis

Baccharis trimera

Aspilia montevidensis Vernonanthura nudifolia

RUBIACEAE

Borreria verticillata

Relbunium hypocarpium

CONVOLVULACEAE

Ipomoea cairica

ONAGRACEAE

Ludwigia longifolia

\section{LYTHRACEAE}

Cuphea glutinosa

Cuphea carthagenensis

VERBENACEAE

Stachytarpheta cayennensis

\section{CLUSIACEAE}

Hypericum sp.

Hypericum brasiliensis

FLACOURTIACEAE

Casearia sylvestris
Insetos visitantes florais

Área

Apis mellifera, Augochlora amphitrite, Augochlora sp. 1, Augochloropsis cupreola, Bombyliidae sp. 2, Callimormus beda, Ceratina (Ceratinula) sp., Cephalurgus anomalus, Melissoptila bonaerensis, Dialictus cf. anisitsianus, Dialictus sp. 1, Diptera sp. 3, Dolichopodidae sp., Dryadula phaetusa, Dryas iulia, Heliconius erato phyllis, Heliopetes alana, H. omrina, Hypalastoroides sp., Idionysson sp., Masarygus sp., Mechanitis lysimnia Montezumia nigriceps, Panurgillus reticulatus, Pyrginae sp. 2, Pyrginae sp. 5, Pyrginae sp.

12, Plebeia droryana, Prionyx thomae, Pseudagapostemon pruinosus, Pseudauglochlora graminea, Ptilothrix relata, Pyrgus orcus, Salpingogaster sp., Sarcophagidae sp., Stenodynerus sp., Tenebrionidae sp., Toxomerus sp. 1, Toxomerus sp. 2, Trina g. geometrina Augochlora amphitrite, Ptilothrix relata

Coelioxys sp., Stenonartonia guaranitica

Agraulis vanillae maculosa, Augochloropsis cupreola, Bibionidae sp., Ceratina

(Ceratinula) sp., Dialictus sp. 2, Dialictus sp. 4, Dryas iulia, Entypus ferruginipennis,

Eristalis sp. 2, Euphoria lurida, Heliconius erato phyllis, Hypancistrocerus sp.,

Megachile sp. 3, Melissoptila bonaerensis, Otitidae sp., Pyrginae sp. 6, Pyrginae sp. 11, Pyrginae sp. 12, Polites vibex catilina, Prionyx thomae, Psaenythia bergi, Psaenythia litoralis, Pyrgus orcus, Salpingogaster sp., Stenodynerus sp., Toxomerus sp. 1, Urbanus teleus, Vehilius s. stictomenes

Anartia amathea roeselia, Apis mellifera, Augochlora sp. 3, Augochloropsis sp. 3, Diptera sp. 1, Dryas iulia, Eristalis sp. 1, Eristalis sp. 2, Euphoria lurida, Heliconius erato phyllis, Omicron aurantiopictum, Polistes cinerascens

Curculionidae sp., Helias phalaenoides palpalis, Lygaeidae sp. 1, Polistes cinerascens Entypus ferruginipennis

Adelpha syma

Augochlora amphitrite, Megachile sp. 2, Psaenythia bergi, Tegosa claudina, Toxomerus sp. 1 Adelpha syma, Augochloropsis sp. 1, Heliconius erato phyllis

RE

$\mathrm{RE}$

EE

$\mathrm{RE} / \mathrm{EE}$

$\mathrm{RE}$

EE

Hypalastoroides sp.

$\mathrm{RE}$

Alphamenes campanulatus, Ancistroceroides sp., Ancistrocerus sp., Anoplius inaurata, Apis mellifera, Augochlora tantilla, Augochloropsis cupreola, Bombyliidae sp. 1, Cerceris sp., Chrysis sp., Diptera sp. 5, Entypus ferruginipennis, Eristalis sp. 2, Eristalis sp. 3, Gorgythion b. begga, Hypancistrocerus sp., Pyrginae sp. 4, Pyrginae sp. 10, Pyrginae sp. 14, Polybia ignobilis, P. sericea, Prionyx thomae, Psaenythia bergi, Pyrgus orcus, Salpingogaster sp., Syrphidae sp., Tachinidae sp.

Montezumia nigriceps

EE

Ancylocelis sp., Arhysoceble picta, Augochlora amphitrite, Augochlora sp. 2, Augochloropsis sp. 2, Bombus atractus, Ceratina cf. asuncionis, C. hyemalis, C. paraguayensis, Masarygus sp., Megacerus reticulatus, Melitoma segmentaria, Odyneropsis sp., Psaenythia bergi, Ptilothrix sp., Zariaspes mys, Zonitolytta chrysomeloides

Augochlora amphitrite, Augochlora sp. 2, Augochlora sp. 4, Augochloropsis cupreola, A. sparsilis, Ceratina $\mathrm{cf}$. asuncionis, Chalepogenus sp., Chrysomelidae sp., Lygaeidae sp. 1, Megachile sp. 4, Melissoptila bonaerensis, M. paraguayensis, Meloidae sp., Hypanthidium divaricatum, Pyrginae sp. 13, Psaenythia bergi, P. litoralis, Pseudagapostemon pruinosus

Chalepogenus sp.

Adelpha syma, Augochlora amphitrite, Callimormus beda, Ceratina sp., Melissoptila sp. 1, Melissoptila sp. 2, Pyrginae sp. 3, Pyrginae sp. 4, Pyrginae sp. 11, Pyrginae sp. 12, Pyrgus orcus, Trina g. geometrina

Chalepogenus sp., Hypanthidium divaricatum, Psaenythia litoralis, Toxomerus sp. 1

EE

Achlyodes mithradates, Agraulis vanillae maculosa, Anartia amathea roeselia, Apis mellifera, Dryas iulia, Gorgythion b. begga, Heliconius erato phyllis, Pyrginae sp. 3, Pyrginae sp. 9, Pyrginae sp. 11, Pyrgus orcus, Salpingogaster sp., Urbanus teleus

Actinote sp., Anartia amathea roeselia, Apis mellifera, Augochloropsis sparsilis, Bombus atractus, Ceratina cf. asuncionis, Chlorion hemipyrrhum, Melissodes sp., Dialictus sp. 3, Plebeia droryana, Polistes actaeon, P. cinerascens, Psaenythia litoralis, Ptilothrix relata Augochlorella michaelis, Dryas iulia, Heliconius erato phyllis

Anthrenoides sp. nov., Bibionidae sp., Cephalurgus anomalus, Diptera sp. 3, Diptera sp. 4, Dryas iulia, Heliconius erato phyllis, Podagritus bocainus, Psaenythia bergi Apis mellifera 
Tabela IV (cont.)

\section{CYPERACEAE}

Eleocharis nudipes

IRIDACEAE

Sysirinchium sp. 2

Herbertia pulchella

Sysirinchium sp. 1

ROSACEAE

Rubus sellowii

FABACEAE/FABOIDAE

Desmodium sp.

\section{OXALIDACEAE}

Oxalis articulata

MELASTOMATACEAE

Tibouchina asperior

Tibouchina gracilis

ACANTHACEAE

Hygrophila brasiliensis

JUNCACEAE

Juncus microcephalus

LAMIACEAE

Hyptis brevipes

HYDROPHYLLACEAE

Hydrolea spinosa

HYPOXIDACEAE

Hypoxis decumbens

Augochlora sp. 2, Dialictus sp. 3, Psaenythia bergi, Stenodynerus sp.

Augochloropsis cupreola, Cephalurgus anomalus, Parapsaenythia sp. nov., Salpingogaster sp., Toxomerus sp. 2, Trichaltica sp.

RE

Apis mellifera, Arawacus separata, Dialictus cf. anisitsianus, Dialictus sp. 3, Entypus ferruginipennis, Pyrginae sp. 2, Pyrginae sp. 11, Pompilidae sp. 3, Stenodynerus sp., Toxomerus sp. 2

Agraulis vanillae maculosa, Augochlora amphitrite, Dialictus cf. anisitsianus, Pyrginae sp. 3

EE

Pentatomidae sp.

Apis mellifera, Augochloropsis sp. 2, Ceratina cf. asuncionis, C. hyemalis, Lygaeidae sp. 1, Lygaeidae sp. 2, Mischocyttarus drewseni, Polistes actaeon, P. cinerascens, Prionyx thomae, Psaenythia bergi

Augochlora amphitrite, Augochlorella michaelis, Buprestidae sp., Ceratina cf. asuncionis, Chalepogenus sp., Megachile sp. 2, Pyrginae sp. 6

Megachile sp.1, Psaenythia bergi

Dialictus cf. anisitsianus

Anthrenoides sp. nov., Ceratina hyemalis, Pyrginae sp. 3, Pseudagapostemon pruinosus Anthrenoides sp. nov., Ceratina (Ceratinula) sp., Hermeuptychia hermes, Panurgillus reticulatus, Pyrginae sp. 7, Pyrginae sp. 8, Pyrginae sp. 14

EE

Buprestidae sp., Macraspis sp., Rutela lineola, Xylocopa frontalis

RE

Augochloropsis sp. 1

$\mathrm{EE}$

Anartia amathea roeselia, Ancistrocerus sp., Paratetrapedia fervida, Polites vibex catilina

Agradecimentos. Aos Drs. Lílian Eggers (UFRGS) e Cláudio Mondin (PUCRS), pela identificação das plantas; à Dra. Birgit Harter (UNESC) e à M.Sc. Kelli Ramos (UFPR), pela identificação das abelhas; à Dra. Helena Romanowski e ao M.Sc. Cristiano Iserhard (UFRGS), pela identificação dos lepidópteros; ao M.Sc. Luciano Moura (FZB-RS), pela identificação dos coleópteros; aos Drs. Bolívar Garcete (Museo Nacional de Historia Natural del Paraguay) e Roberto Alejandro Cambra (Universidad de Panama), pela identificação das vespas; ao Dr. Carlos José Einicker Lamas (MZUSP), pela identificação dos dípteros e a todos que direta ou indiretamente contribuíram na realização deste trabalho.

\section{REFERÊNCIAS BIBLIOGRÁFICAS}

Aguiar, A. J. C. \& Martins, C. F. 2003. The bee diversity of the Tabuleiro vegetation in the Guaribas Biological Reserve (Mamanguape, Paraíba, Brazil). In: Melo, G. A. R. \& AlvesDos-Santos, I. eds. Apoidea Neotropica: homenagem aos 90 Anos de Jesus Santiago Moure. Criciúma, UNESC. p.209-216.

Aizen, M. A. \& Feinsinger, P. 1994. Forest fragmentation, pollination, and plant reproduction in a chaco dry forest, Argentina. Ecology 75(2):330-351.

Alves-Dos-SAntos, I. 1999. Abelhas e plantas melíferas da mata atlântica, restinga e dunas do litoral norte do estado do Rio Grande do Sul, Brasil. Revista Brasileira de Entomologia 43(3/4):191-223.
Antonini, Y. \& Martins, P. R. 2003. The flowering-vistiting bees at the ecological station of the Universidade Federal de Minas Gerais, Belo Horizonte, MG, Brazil. Neotropical Entomology 32(4):565-575.

Barbola, I. F. \& Laroca, S. A. 1993. Comunidade de Apoidea (Hymenoptera) da Reserva Passa Dois (Lapa, Paraná, Brasil): I. Diversidade, abundância relativa e atividade sazonal. Acta Biológica Paranaense 22(1-4):91-113.

Bosch, J.; Renata, J. \& Cerdá, X. 1997. Flowering phenology, floral traits and pollinator composition in a herbaceous Mediterranean plant community. Oecologia 109:583-591.

Brown JR., K. S. 1997. Diversity, disturbance and sustainable use of Neotropical forests: insects as indicators for conservation monitoring. Journal of Insect Conservation 1(1):25-42.

Brown JR., K. S. \& Freitas, A. V. L. 2002. Butterfly communities of urban Forest fragments in Campinas, São Paulo, Brazil: structure, instability, environmental correlates, and conservation. Journal of Insect Conservation 6(4):217-231.

BudDle, C. M. 2001. Spiders (Araneae) associated with downed woody material in a deciduous forest in central Alberta, Canada. Agricultural and Forest Entomology 3(4):241-251.

Chey, V. K.; Holloway, J. D.; Hambler, C. \& Speight, M. R. 1998. Canopy knockdown of arthropods in exotic plantations and natural Forest in Sabah, north-east Borneo, using insecticidal mist-blowing. Bulletin of Entomological Research 88(1):15-24.

Comba, L.; Corbet, S. A.; Hunt, L. \& Warren, B. 1999. Flowers, nectar and insect visits: evaluating british plant species for 
pollinator-friendly gardens. Annals of Botany 83:369-383.

Cure, J. R.; Thiengo, M.; Silveira, F. A. \& Rocha, L. B. 1992. Levantamento da fauna de abelhas silvestres na "Zona da Mata" de Minas Gerais. III. Mata Secundária na região de Viçosa (Hymenoptera, Apoidea). Revista Brasileira de Zoologia 9(3,4):223-239.

Del Moral, R. \& Muller, C. H. 1969. Fog drip: a mechanism of toxin transport from Eucalyptus globulus. Bulletin of the Torrey Botanical Club 96(4):467-475.

Denys, C. \& Tscharntke, T. 2002. Plant-insect communities and predator-prey ratios in field margin strips, adjacent crop fields, and fallows. Oecologia 130(2):315-324.

Didham, R. K.; Ghazoul, J.; Stork, N. E. \& Davis, A. J. 1996. Insects in fragmented forests: a functional approach. Trends in Ecology and Evolution 11(6):255-260.

Ferraro, L. W. \& Hasenack, H. 2000. Clima. In: Carvão e meio ambiente. Porto Alegre, UFRGS. p.22-44.

FriebE, B. 1983. Zur Biologie eines Buchenwaldbodens: 3. Die Kaferfauna 41:45-80.

Fontana, S.; Bencke, G. A. \& Reis, R. E. orgs. 2003. Livro vermelho da fauna ameaçada de extinção no Rio Grande do Sul. Porto Alegre, EDIPUCRS. 632p.

Gaglianone, M. C. 2000. Biologia floral de espécies simpátricas de Malvaceae e suas abelhas visitantes. Biociências 8(1):13-31.

Golden, D. M. \& CRist, T. O. 1999. Experimental effects of habitat fragmentation on old-field canopy insects: community, guild and species responses. Oecologia 118(3):371-380.

GotTsberger, G. 1986. Some pollination strategies in neotropical savannas and forests. Plant Systematics and Evolution 152(1,2):29-45.

Hammer, O. \& Harper, D. A. T. 2003. PAST, version 1.11 Disponível em: 〈http://folk.uio.no/ohammer/past〉. Acesso em: 20.07.2004

Harter, B. 1999. Bienen und ihre Trachtpflanzen im Araukarien- Hochland von Rio Grande do Sul, mit Fallstudien zur Bestäubung von Pionierpflanzen. Tübingen Eberhar d-Karls Universität. 185p.

Hingston, A. B. \& PotTs, B. M. 1998. Floral visitors of Eucalyptus globulus subsp. globulus in eastern Tasmania. Tasforests 10:125-139.

Huston, M. A. 1979. A general hypothesis of species diversity. American Naturalist 113(1):81-101

Hutcheson, J. 1999. Characteristics of Mapara insect communities as depicted by Malaise trapped beetles: changes with time and animal control. Science for conservation 135:1-20.

Huttel, C. \& Loumeto, J.J. 2001. Effect of exotic tree plantations and site management on plant Diversity. In: BERnHARDReversat, F. ed. Effect of exotic tree plantations on plant diversity and biological soil fertility in the Congo savanna: with special reference to eucalypts. Center for International Forestry Research, Bogor, Indonesia. p.9-18.

IUCN. 2002. 2002 red list of threatened species. The IUCN species survival commission. Disponível em: <http:// www.redlist.org > Acesso em: 20.09.2004.

Jolivet, P. 1992. Insects and plants, parallel evolution and adaptations. 2 ed. Florida, Sandhill Crane. 190p.

Kearns, C. A. \& Inouye, D. W. 1997. Pollinators, flowering plants, and conservation biology. BioScience 47(5):297-397.

Kearns, C. A.; Inouye, D. W. \& Waser, N. M. 1998. Endangered mutualisms: the conservation of plant-pollinator interactions. Annual Review of Ecology and Systematics 29:83-112.

Krebs, C. J. 1998. Ecological methodology. Menlo Park, Benjamin/ Cummings. 581p.

Kremen, C.; Colwell, R. K.; Erwin, T. L.; Murphy, D. D.; Noss, R. F. \& SANJAYAN, M. A. 1993. Terrestrial arthropod assemblages: their use in conservation planning. Conservation Biology 7(4):796-808.

Kruess, A. \& TscharntKe, T. 1994. Habitat fragmentation, species loss, and biological control. Science 264(5165):1581-1587.

Lara, F. M. 1992. Princípios de entomologia. Jaboticabal, UNESP. 278p.

MagurRan, A. E. 1988. Ecological diversity and its measurement. New Jersey, Princeton University Press. 179p.

Majer, J. D. \& Recher, H. F. 1999. Are eucalypts Brazil's friend or foe? An entomological viewpoint. Anais da Sociedade Entomológica do Brasil 28(2):185-200.
Marinoni, R. C. \& Dutra, R. R. C. 1991. Levantamento da fauna entomológica no estado do Paraná. I. Introdução. Situações climática e florística de oito pontos de coleta. Dados faunísticos de agosto de 1986 a julho de 1987. Revista Brasileira de Zoologia 8(1-4):31-73.

McAleece, N. 1997. Biodiversity Professional Beta 1.0. Versão 1.0. The Natural History Museum and the Scottish Association for Marine Science. Disponível em: <http:// www.nhm.ac.uk/zoology/bdpro>. Acesso em: 20.07.2004.

Morales, C. L. \& Aizen, M. A. 2002. Does invasion of exotic plants promote invasion of exotic flowers visitors? A case study from the temperate forests of the southern Andes. Biological Invasions 4(1,2):87-100.

Oliveira, H. G.; Zanuncio, T. V.; Zanuncio, J. C. \& Santos, G. P. 2001. Coleópteros associados à eucaliptocultura na região de Nova Era, Minas Gerais, Brasil. Floresta e Ambiente 8(1):52-60.

Pascarella, J. B.; Waddington, K. D. \& Neal, P.R. 2001. Nonapoid flower-visiting fauna of Everglades National Park, Florida. Biodiversity and Conservation 10:551-566.

Pickett, S. T. A.; Ostfeld, R. S.; Shachak, M. \& Likens, G. E. 1997. The ecological basis of conservation. Heterogeneity, ecosystems, and biodiversity. New York, Chapman \& Hall. 466p.

Pinto, R.; Junior, J. S. Z.; Zanuncio, T. V.; Zanuncio, J. C. \& LACERDA, M. C. 2004. Coleópteros coletados com armadilhas luminosas em plantios de Eucalyptus urophylla na região amazônica brasileira. Ciência Florestal 14(1):111-119.

Poggiani, F. \& Oliveira, R. E. 1998. Indicadores para conservação dos núcleos de vida silvestre. Série Técnica do Instituto de Pesquisas e Estudos Florestais 12(31):45-52.

Rамво, B. 1994. A fisionomia do Rio Grande do Sul: ensaio de monografia natural. São Leopoldo, UNISINOS. 473p.

SaKagami, S. F.; Laroca, S. \& Moure, J. S. 1967. Wild bee biocoenotics in São José dos Pinhais (PR), South Brazil. Preliminary Report. Journal of the Faculty of Science Hokkaido University Serie VI, Zoology 16(2):253-291.

SAMWAYS, M. J. 1995. Insect conservation biology. London, Chapman \& Hall. 358p.

Santos, G. P.; Zanuncio, T. V.; Vinha, E. \& Zanuncio, J. C. 2002. Influência de faixas de vegetação nativa em povoamentos de Eucalyptus cloeziana sobre população de Oxydia vesulia (Lepidoptera: Geometridae). Revista Árvore 4(4):499-504.

Schlindwein, C. 1995. Wildbienen und ihre Trachtpflanzen in einer südbrasilianischen Buschlanschaft: Fallstudie Guaritas, bestäubung bei Kakteen und Loasaceen. Stuttgart, Ulrich E. Grauer. 141p.

Seastedt, T. R. \& Crossley, D. A. 1984. The influence of arthropods on ecosystems. BioScience 34:157-161.

Silveira Neto, S.; Monteiro, R.; Zucchi, R. C. \& Morais, R. C. B 1995. Uso da análise faunística de insetos na avaliação do impacto ambiental. Scientia Agricola 52(1):9-15.

Silveira Neto, S.; Nakano, O.; Barbin, D. \& Nova, N. A. V. 1976. Manual de ecologia dos insetos. São Paulo, CERES. 419p.

Souto, X. C.; Bolaño, J. C.; González, L. \& Reigosa, M. J. 2001. Allelopathic effects of tree species on some soil microbial populations and herbaceous plants. Biologia Plantarum 44(2):269-275.

Speight, M. R.; Hunter, M. D. \& Watt, A. D. 1999. Ecology of insects concepts and applications. Oxford, Blackwell Science. $350 \mathrm{p}$

Strahler, A. N. 1977. Geografia física. Barcelona, Omega, 2. ed. $767 p$.

Taura, H. M. \& Laroca, S. 2001. A associação de abelhas silvestres de um biótopo urbano de Curitiba (Brasil), com comparações espaço-temporais: abundância relativa, fenologia, diversidade e exploração de recursos (Hymenoptera, Apoida). Acta Biológica Paranaense 30(1-4):35-137.

Teixeira, M. B.; Coura-Neto, A. B.; Pastore, U. \& Rangel Filho, A. L. R. 1986. Vegetação; as regiões fitoecológicas, sua natureza, seus recursos econômicos; estudo fitogeográfico. In: Levantamento de recursos naturais. Instituto Brasileiro de Geografia e Estatística, Rio de Janeiro, v. 33, p.541-632.

Tepedino, V. J. 1979. The importance of bees and other insect pollinators in maintaining floral species composition. Great Basin Naturalist Memoirs 3:139-150. 
Tscharntke, T. A. \& Brandl, R. 2004. Plant-Insect interactions in fragmented landscapes. Annual Review of Entomology 49:405-430.

Tscharntke, T. A.; Dewenter, I. S.; Kruess, A. \& Thies, C. 2002. Characteristics of insect populations on habitat fragments: a mini review. Ecological Research 17:229-239.

Viana, V. M. \& Pinheiro, L. A. F. V. 1998. Conservação da biodiversidade em fragmentos florestais. Série Técnica do Instituto de Pesquisas e Estudos Florestais 12(32):25-42.

WASER, N. M. 1978. Interspecific pollen transfer and competition between co-occurring plant species. Oecologia 36(2):223-236.

Wilms, W.; Imperatriz-Fonseca, V. L. \& Engels, W. 1996. Resource Partitioning between highly eusocial bees and possible impact of the introduced Africanized honey bee on native stingless bees in the Brazilian Atlantic Rainforest. Studies on Neotropical Fauna and Environment 31:137-151.

Wilms, W.; Wendel, L.; Zillikens, A.; Blochtein, B. \& Engels, W. 1997. Bees and other insects recorded on flowering trees in a subtropical Araucaria forest in southern Brazil. Studies on Neotropical Fauna and Environment 32:220-226.

Wittmann, D. \& Hoffmann, M. 1990. Bees of Rio Grande do Sul, southern Brazil (Insecta, Hymenoptera, Apoidea). Iheringia, Série Zoologia, (70):17-43.

Zanella, F. C. V. 2000. The bees of the caatinga (Hymenoptera, Apoidea, Apiformes): a species list and comparatives notes regarding their distribution. Apidologie 31:579-592.

Recebido em novembro de 2005. Aceito em novembro de 2006. ISSN 0073-4721

Artigo disponível em: www.scielo.br/isz 